\title{
Antedrug Budesonide by Intrapulmonary Treatment Attenuates Bleomycin-Induced Lung Injury in Rats with Minimal Systemic Adverse Effects
}

\author{
Masayuki Kohno, ${ }^{a}$ Mari Haramoto,,${ }^{a}$ Oumi Nakajima, ${ }^{a}$ Liying Yang, ${ }^{a}$ Shiro Hinotsu, ${ }^{a}$ \\ Masanao YoKohiRa, ${ }^{b}$ Katsumi Imaida, ${ }^{b}$ and Koji KaWAKAMI ${ }^{*, a}$ \\ ${ }^{a}$ Department of Pharmacoepidemiology, Graduate School of Medicine and Public Health, Kyoto University; \\ Yoshidakonoecho, Sakyo-ku, Kyoto 606-8501, Japan: and ${ }^{b}$ Onco-Pathology, Department of Pathology and Host-Defense, \\ Faculty of Medicine, Kagawa University; 1750-1 Ikenobe, Miki-cho, Kita-gun, Kagawa 761-0793, Japan. \\ Received March 23, 2010; accepted April 25, 2010; published online April 27, 2010
}

\begin{abstract}
Corticosteroids are routinely used in patients with pulmonary fibrosis, yet they have several adverse effects. To improve this situation, we used an animal model of pulmonary injury and early fibrosis and investigated whether the combination of an intrapulmonary inhalation device with antedrug budesonide (BUD) administered to the lung had greater efficacy and fewer systemic adverse effects compared to long-acting dexamethasone (DEX). BUD or DEX was administrated either intrapulmonary or intravenously to bleomycin-treated rats. Antiinflammatory and antifibrotic effects were evaluated according to inflammatory cell count, total protein concentration and soluble collagen concentration in bronchoalveolar lavage fluid. The systemic immunosuppressive effects were also assessed by measuring body, spleen and thymus weight. BUD and DEX were compared with respect to their pharmacokinetic profiles in plasma and lung. Intrapulmonary treatment of BUD attenuates various inflammatory and early fibrotic indices with minimal systemic adverse effects compared with DEX. The area under the curve $(A U C)$ of BUD by intrapulmonary spray was 6.6-fold higher than the $A U C$ of DEX in the lung. This study suggests that antedrug BUD by intrapulmonary treatment has local anti-inflammatory and antifibrotic effects with minimal systemic adverse effects.
\end{abstract}

Key words antedrug; intrapulmonary treatment; corticosteroid; drug delivery

Idiopathic pulmonary fibrosis (IPF) is a progressive and life-threatening disease. It is characterized by accumulation of inflammatory cells, fibroblast proliferation and accumulation of extracellular matrix in the alveolar and interstitial spaces. ${ }^{1)}$ The current and most conventional therapy for IPF involves treatment with corticosteroids, other immunosuppressive agents and antifibrotic agents. Recently, there have been many clinical trials of novel therapies for IPF. However, the results have mostly been disappointing. ${ }^{2,3)}$

Corticosteroids are usually administrated in an attempt to control the inflammation in fibrotic lungs, but - in addition to their efficacy being not well established - they cause various adverse reactions. ${ }^{4)}$ "Antedrugs" are administered locally, and possess local specific activity, but are rapidly metabolized to inactive compounds into the circulation, resulting in no systemic toxicity. ${ }^{5)}$ Budesonide (BUD), an inhaled corticosteroid antedrug possesses local specific anti-inflammatory activity and is effective for asthma, rhinitis and inflammatory bowel diseases. $^{6-8)}$

The aim of this study was to clarify whether intrapulmonary treatment with BUD attenuates bleomycin (BLM)induced lung injury and early fibrosis with minimal adverse effects in rats and whether the antedrug BUD has a superior risk/benefit balance to the traditional corticosteroid dexamethasone (DEX).

\section{MATERIALS AND METHODS}

Reagents BLM was purchased from Nippon Kayaku (Tokyo, Japan). BUD and DEX (dexamethasone 21-phosphate disodium salt) were purchased from Sigma (St. Louis, MO, U.S.A.). Protein assay Coomassie Brilliant Blue (CBB) solution was purchased from Nacalai Tesque (Kyoto, Japan).
Sircol soluble collagen assay kit was purchased from Biocolor (Belfast, Northern Ireland). Lactate dehydrogenase (LDH) detection kit was purchased from Roche Diagnostics (Mannheim, Germany).

Animals Female Sprague-Dawley rats (weight 180 $220 \mathrm{~g}$ ) purchased from SLC Japan, Inc. (Hamamatsu, Japan) were used in this study. Animals were housed under constant temperature $\left(24 \pm 2{ }^{\circ} \mathrm{C}\right)$ and humidity $(50 \pm 10 \%)$ with a controlled $12 \mathrm{~h}$ light/dark cycle. All animal experiments were carried out in accordance with the guidelines of the Kyoto University School of Medicine.

Intrapulmonary Spray Administration Intrapulmonary spray (i.s.) administrations were performed using an IA-1C-R Microsprayer ${ }^{\mathrm{TM}}$ (Penn-Century, Philadelphia, U.S.A.) connected to a FMJ-250 high-pressure syringe (Penn-Century) containing $100 \mu \mathrm{l}$ of the solution. Under a general anesthesia with isoflurane, the tip of the microsprayer was introduced into the rat's trachea using a fiber optic light. ${ }^{9,10)}$

Animal Studies On day 0, rats received a single intrapulmonary spray $(100 \mu \mathrm{l})$ of saline containing BLM $(5 \mathrm{mg} / \mathrm{kg})$ or saline alone (sham group only). BUD or DEX dissolved in saline or saline alone was administrated by intrapulmonary spray or intravenous (i.v.) injection on days 1,3 and 5 (Table 1). On day 7, a laparotomy was performed and blood was drawn from the abdominal aorta under isoflurane anesthesia, after which the animals were killed, and the lung promptly removed and weighed. Also on day 7 , we evaluated the severity of lung inflammation by bronchoalveolar lavage fluid (BALF) analysis and the effect of BUD and DEX on pulmonary injury and early fibrosis by histological evaluation according to the alveolitis score of the lungs.

Cell Counts and Biochemical Studies in BALF BALF 
Table 1. Study Protocol

\begin{tabular}{|c|c|c|c|c|c|c|c|}
\hline \multirow{2}{*}{ Group } & \multicolumn{3}{|c|}{ Day 0} & \multicolumn{3}{|c|}{ Day 1,3 , and 5} & \multirow{2}{*}{$\begin{array}{c}\text { Day } 7 \\
\text { Sacrifice }\end{array}$} \\
\hline & Inducer & Dose & Route & Treatment & Dose & Route & \\
\hline \multicolumn{8}{|c|}{ Experiment 1 (effects of DEX, Figs. 1, 2) } \\
\hline Sham & Saline & - & i.s. & - & - & - & $\bigcirc$ \\
\hline $\mathrm{BLM}+$ saline & BLM & $5 \mathrm{mg} / \mathrm{kg}$ & i.s. & Saline & - & i.s. & $\bigcirc$ \\
\hline $\mathrm{BLM}+\mathrm{DEX}$ & BLM & $5 \mathrm{mg} / \mathrm{kg}$ & i.s. & DEX & $1 \mathrm{mg} / \mathrm{kg}$ & i.s. & $\bigcirc$ \\
\hline \multicolumn{8}{|c|}{ Experiment 2 (effects of DEX vs. BUD, Figs. 3, 4) } \\
\hline Sham & Saline & - & i.s. & - & - & - & $\bigcirc$ \\
\hline BLM+saline & BLM & $5 \mathrm{mg} / \mathrm{kg}$ & i.s. & Saline & - & i.s. & 0 \\
\hline BLM+DEX i.s. & BLM & $5 \mathrm{mg} / \mathrm{kg}$ & i.s. & DEX & $0.2 \mathrm{mg} / \mathrm{kg}$ & i.s. & $\bigcirc$ \\
\hline BLM+BUD i.s. & BLM & $5 \mathrm{mg} / \mathrm{kg}$ & i.s. & BUD & $0.2 \mathrm{mg} / \mathrm{kg}$ & i.s. & 0 \\
\hline BLM+DEX i.v. & BLM & $5 \mathrm{mg} / \mathrm{kg}$ & i.s. & DEX & $0.2 \mathrm{mg} / \mathrm{kg}$ & i.v. & 0 \\
\hline
\end{tabular}

was obtained by washing the lung twice times with $2.5 \mathrm{ml}$ aliquots of phosphate buffered saline (PBS) through a tracheal cannula. The BALF was centrifuged $(300 \boldsymbol{g}, 5 \mathrm{~min}$, $20^{\circ} \mathrm{C}$ ) and the cell pellet was resuspended and counted using a haemocytometer. The cell-free supernatant was used for biochemical determinations. Total protein concentration was measured by protein assay $\mathrm{CBB}$ solution. LDH activity and soluble collagen concentration were measured using commercial kits.

Histological Analysis Lung was fixed in 4\% buffered formaldehyde and paraffin-embedded $5 \mu \mathrm{m}$ sections were stained with hematoxylin and eosin. The images were captured using a BX-51 microscope equipped with a DP-25 CCD camera (Olympus, Tokyo, Japan). The severity of alveolitis was blindly assessed semi-quantitatively, according to the criteria previously described by Yao et al. ${ }^{11)}$

Corticosteroid Detection by High-Performance Liquid Chromatography (HPLC) Bud or DEX was administrated to rats via i.v. or i.s. routes as a bolus dose of $1 \mathrm{mg} / \mathrm{kg}$. The rats were sacrificed $0.5,1,3$, and $6 \mathrm{~h}$ after start of administration (three animals at each time point). Plasma and lung were collected at all time points. The amount of BUD or DEX in plasma or lung homogenate was determined by modifying a method that had been described previously. ${ }^{12,13)}$ Lung treated with DEX was homogenized in $1 \mathrm{~mm} \mathrm{NaOH}$. The homogenate was shaken in ethyl acetate, centrifuged and the organic layer was collected and dried. Plasma samples treated with DEX were extracted with $1 \mathrm{~mm} \mathrm{NaOH}$. In the case of BUD, plasma samples were extracted with $0.5 \mathrm{~N} \mathrm{HCl}$. Similar procedures to those used for DEX were used for quantification of BUD. Lung treated with BUD was homogenized in $0.9 \%$ saline solution at $\mathrm{pH} 5.0$ prepared with $1 \% 0.5 \mathrm{~N} \mathrm{HCl}$ instead of $1 \mathrm{~mm} \mathrm{NaOH}$. The resultant samples $(10 \mu \mathrm{l})$ were injected onto an HPLC system (GL-7410, GL Sciences, Tokyo, Japan) with a variable-wavelength UV detector (GL7451, GL Sciences) to detect corticosteroid levels. BUD or DEX concentrations are expressed in micro molar, assuming a tissue density of $1 \mathrm{~g} / \mathrm{ml}$.

Statistical Analysis All values are expressed as the mean \pm S.E. and statistical analysis was designed to test for differences using one-way ANOVA as a primary comparison. Post-hoc analysis was performed with Dunnett's procedure. Differences were considered to be significant at $p<0.05$.

\section{RESULTS}

Intrapulmonary Treatment with DEX Attenuates BLM-Induced Lung Injury and Fibrosis To test the ability of DEX to attenuate BLM-induced lung injury and fibrosis when administered intrapulmonally, rats received an intrapulmonary spray of BLM or saline on day 0 , followed by an intrapulmonary spray of DEX $(1 \mathrm{mg} / \mathrm{kg})$ or saline on days 1 , 3 and 5. On day 7 the rats were evaluated (Table 1). Total cell numbers, soluble collagen and total protein in BALF were increased in BLM-sprayed rats compared with saline-sprayed rats. As shown in Fig. 1A, intrapulmonary treatment with DEX reduced total cell numbers, soluble collagen and total protein in BALF compared with saline-treated rats. Increased total protein concentration in BALF is an important marker of damage to the alveolar-capillary barrier of lung. ${ }^{14)}$ Soluble collagen in BALF or lung tissue is one of the markers of early-fibrosis. ${ }^{15,16)}$ Histological examination showed that intrapulmonary treatment with DEX suppressed the infiltration of inflammatory cells into the alveoli and interstitium in the lung (Figs. 1B, C).

Intrapulmonary Treatment with DEX Induces Undesirable Systemic Effects Steroids suppress various inflammatory symptoms but also induce various side effects. Figure 2 represents the undesirable effects of DEX $(1 \mathrm{mg} / \mathrm{kg})$ on spleen and thymus weights in normal, bleomycin-induced and DEX treated rats. Body weights, spleen/body and thymus/body weight ratios were decreased in DEX-treated rats compared with saline-treated rats. Also, white blood cell counts were decreased in DEX-treated rats compared with saline-treated animals (data not shown). These results suggest that intrapulmonary treatment with DEX induces the undesirable systemic symptoms.

Effect of BUD or DEX on Body, Spleen, Thymus and Lung Weights of Rats with BLM-Induced Lung Injury and Fibrosis It has been reported that BUD antedrug shows few side effects compared with other steroids. Therefore, intrapulmonary treatment with BUD would be expected to result in decreased undesirable systemic indices in the BLM model compared with DEX-treated rats. Rats received an intrapulmonary spray of BLM or saline on day 0 , followed by a treatment of BUD $(0.2 \mathrm{mg} / \mathrm{kg}$, i.s. $), \operatorname{DEX}(0.2 \mathrm{mg} / \mathrm{kg}$, i.s. or i.v. routes) or saline (i.s.) on days 1,3 and 5. On day 7 the rats were evaluated (Table 1). Body weights, and spleen/body and thymus/body weight ratios, were decreased in DEX- 

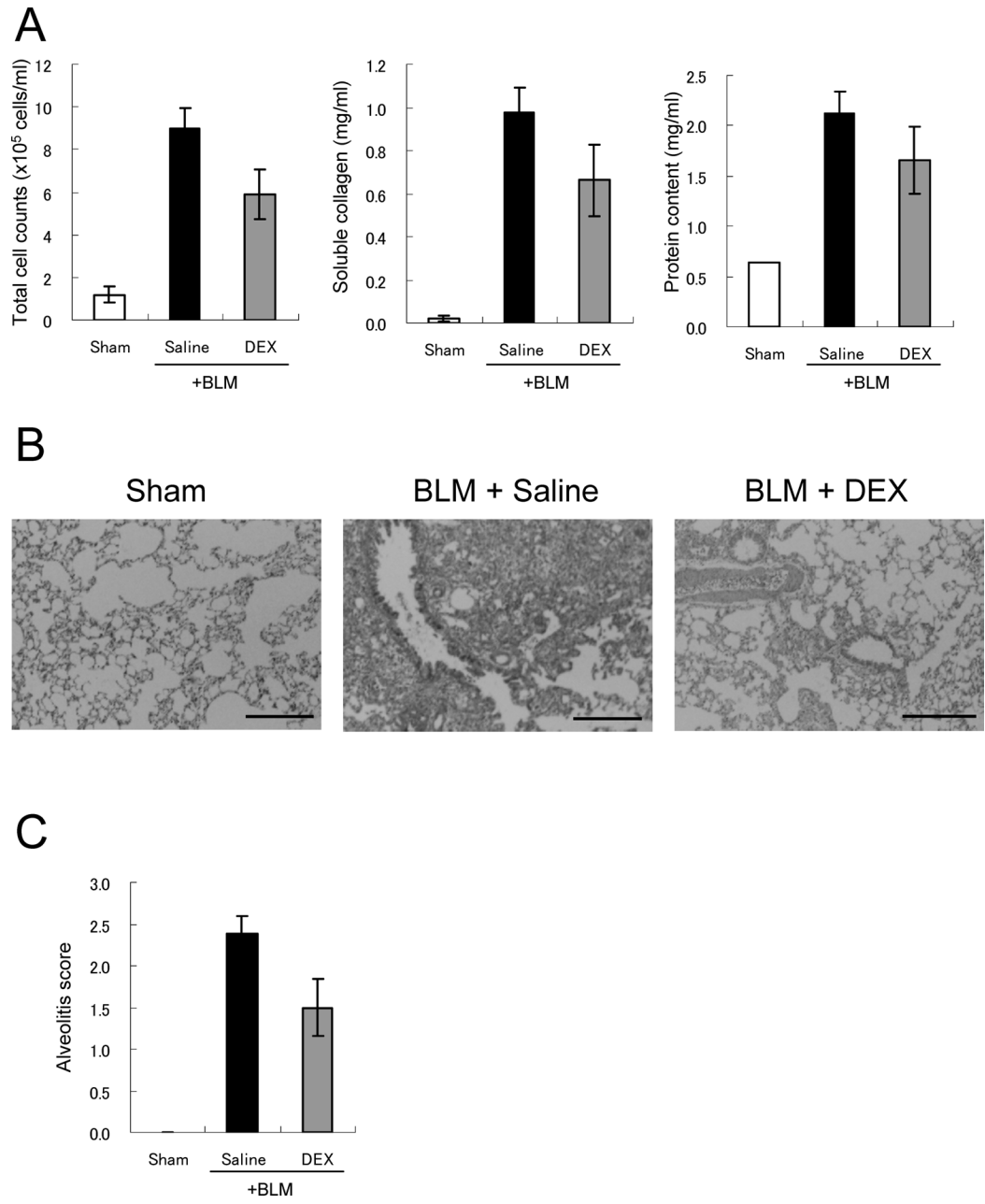

Fig. 1. Intrapulmonary Treatment with DEX Attenuates BLM-Induced Lung Injury and Fibrosis

(A) Effect of DEX on the count of total cells (left), the levels of soluble collagen (middle) and total protein (right) in BALF obtained from rats with BLM-induced lung injury and fibrosis on day 7 after BLM induction. DEX was administered at $1 \mathrm{mg} / \mathrm{kg}$ on days 1,3 and 5 after BLM induction. Data are means \pm S.E. for groups of six animals. (B) Representative photomicrographs of lung histopathology in the sham group (left), BLM+saline (middle) and BLM+DEX (right) $7 \mathrm{~d}$ after BLM induction. Lung sections were stained with hematoxylin and eosin. Scale bar $=200 \mu \mathrm{m}$. (C) Semi-quantitative evaluation of the histological findings by scoring alveolitis change.
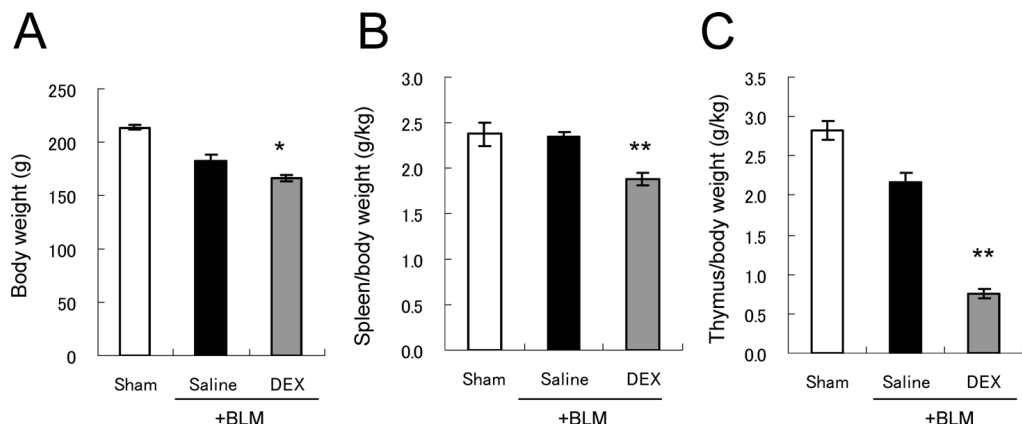

Fig. 2. Intrapulmonary Treatment with DEX Induces Toxic Effects

DEX ( $1 \mathrm{mg} / \mathrm{kg})$ was administered on days 1,3 and 5 after BLM induction, and body (A), spleen (B) and thymus (C) weights were assessed on day 7 . Data are means \pm S.E. for groups of six animals. $* * p<0.01, * p<0.05$ versus the BLM-treated saline group.

treated rats when compared with BUD-treated rats (Figs. $3 \mathrm{~A}-\mathrm{C}$ ). Intrapulmonary treatment with BUD also attenuated the undesirable systemic symptoms: thymus/body weight ratio was less affected in BUD-treated rats compared with DEX-treated rats (Fig. 3C), but the changes were minimal in term of body weight (Fig. 3A) and spleen/body weight ratios (Fig. 3B). In contrast, lung/body weight ratio was decreased in BUD-treated rats compared with DEX-treated rats (Fig.
3D). These results indicate that intrapulmonary treatment with BUD is less toxic than treatment with DEX in BLMinduced rats at a dose of $0.2 \mathrm{mg} / \mathrm{kg}$.

Effect of Corticosteroids on the BALF-Specific Parameters of BLM-Induced Lung Injury and Fibrosis in Rats To test the ability of intrapulmonary treatment with BUD to attenuate BLM-induced lung injury and fibrosis, BALF was evaluated. Total cell numbers, soluble collagen, 

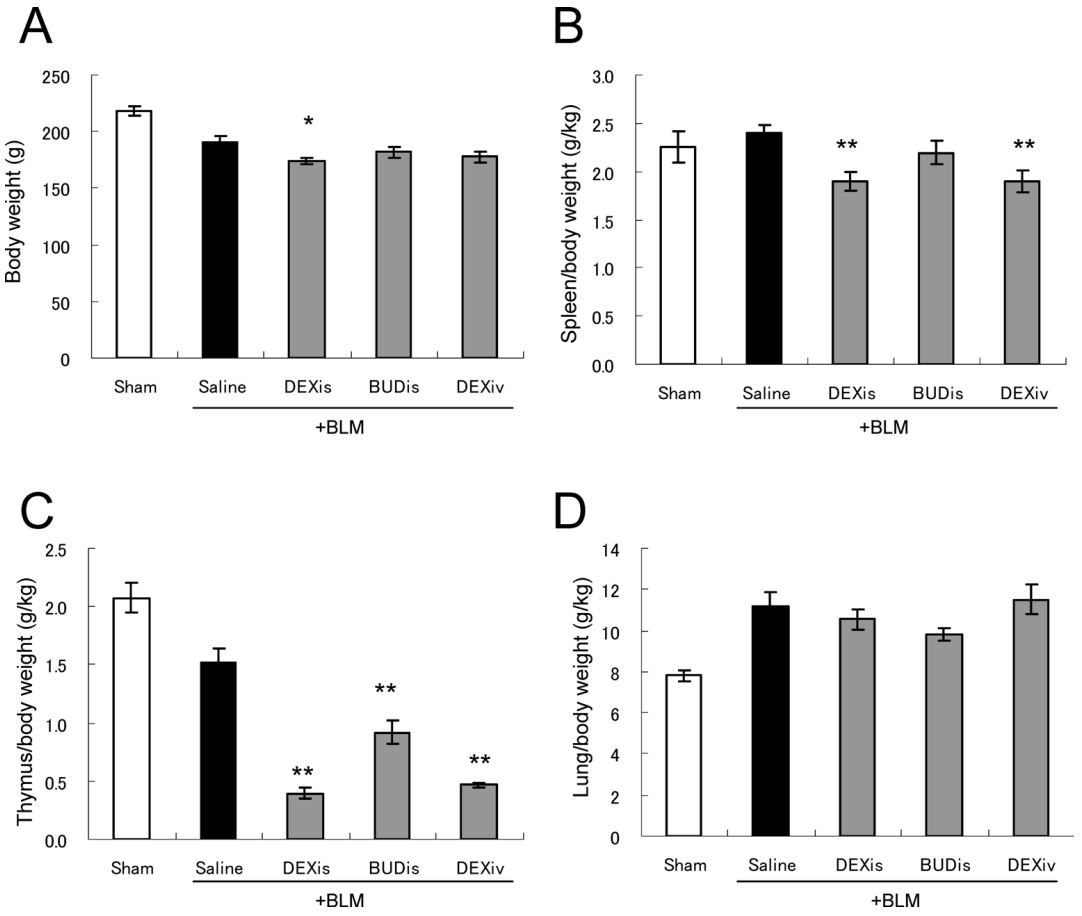

Fig. 3. Effect of BUD and DEX on Body, Lung, Spleen and Thymus Weights of Rats with BLM-Induced Lung Injury and Fibrosis

Ether BUD or DEX was administrated at $0.2 \mathrm{mg} / \mathrm{kg}$ on days 1,3 and 5 after BLM induction, and body (A), spleen (B), thymus (C) and lung (D) weights were assessed on day 7 . Data are means \pm S.E. for groups of six to eight animals. $* * p<0.01, * p<0.05$ versus the BLM-treated saline group. i.s., intrapulmonary spray; i.v., intravenous.

A

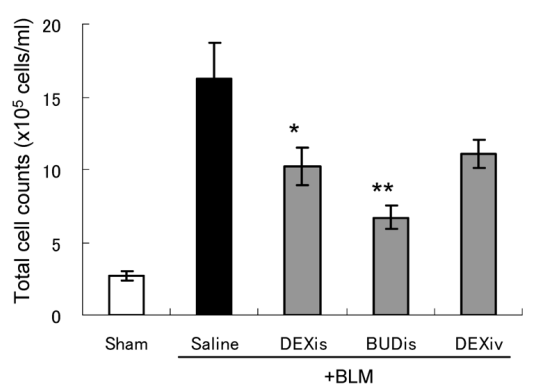

C

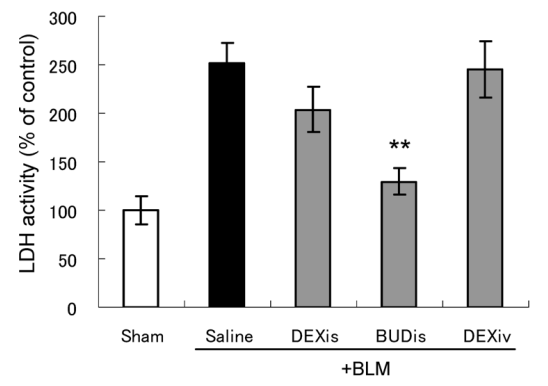

B

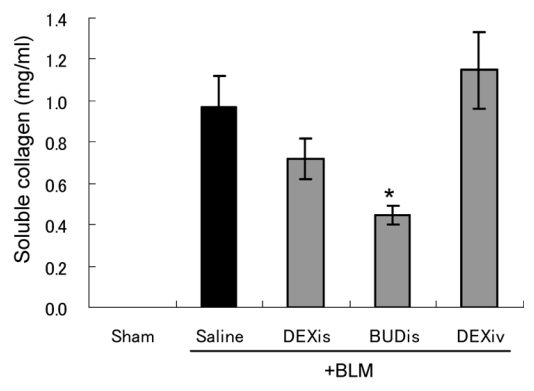

D

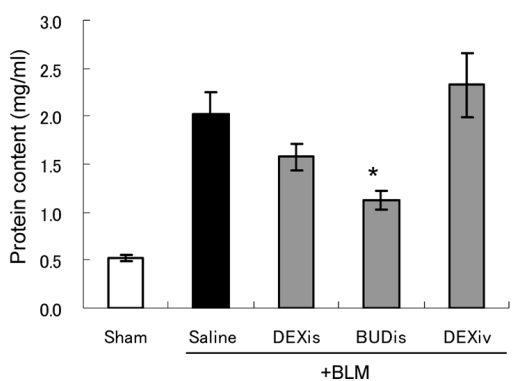

Fig. 4. Effect of BUD and DEX on Total Cells Count (A) and Levels of Soluble Collagen (B), LDH (C) and Total Protein (D) in BALF of Rats with Lung Injury and Fibrosis on Day 7 after BLM Induction

Data are means \pm S.E. for groups of six to eight animals. $* * p<0.01, * p<0.05$ versus the BLM-treated saline group. i.s., intrapulmonary spray; i.v., intravenous.

LDH and total protein in BALF were increased in BLM-induced rats compared with saline control rats, and all the parameters were significantly decreased in BUD-treated rats compared with DEX-treated rats (Fig. 4). LDH activity in BALF represents the extent of damage to lung cells and a general marker of cell injury. ${ }^{14)}$ In addition, intrapulmonary treatment with BUD (twice a week for 2 weeks) was less toxic and more effective than treatment with DEX in BLMinduced rats on day14 (data not shown). These results suggest that intrapulmonary treatment with BUD is superior to that with DEX in term of immunosuppressive efficacy at a dose of $0.2 \mathrm{mg} / \mathrm{kg}$. 
Pharmacokinetic Profiles of BUD and DEX by Intrapulmonary or i.v. Treatment Finally, to assess the difference between the pharmacokinetic profiles of BUD and DEX in blood and lung, rats were given an intrapulmonary spray or i.v. injections of DEX or BUD at a dose of $1 \mathrm{mg} / \mathrm{kg}$. Subsequently, the time-course changes in the plasma and lung levels of DEX or BUD were determined. As shown in Fig. 5a, BUD exhibited improved drug levels in lung, followed by slower clearance from lung when compared with DEX. It was clear that BUD remained in lung tissue for more than $3 \mathrm{~h}$ after spraying, whereas DEX disappeared after $3 \mathrm{~h}$. The area under the curve $(A U C)$ of BUD was 6.6-fold higher than the $A U C$ of DEX in lung (Table 2). On the other hand, DEX was present in the plasma $6 \mathrm{~h}$ after intrapulmonary spraying and its elimination half-life was $4.2 \mathrm{~h}$, whereas BUD was not detectable from $30 \mathrm{~min}$ up to $6 \mathrm{~h}$ (Fig. 5B, Table 2).
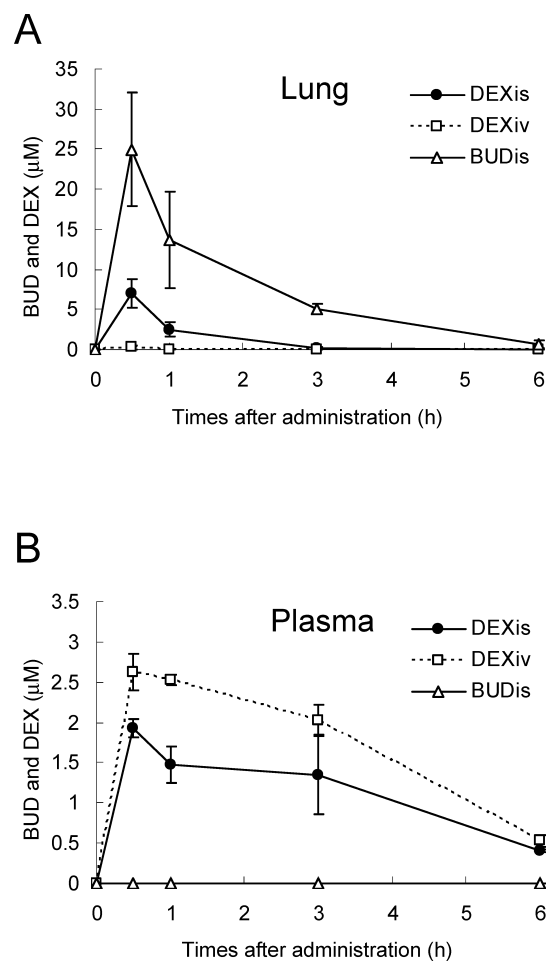

Fig. 5. Lung and Plasma Concentrations of BUD and DEX after Intrapulmonary Spray (i.s.) or Intravenous (i.v.) Treatment

Rats were given BUD or DEX at a dose of $1 \mathrm{mg} / \mathrm{kg}$ by intrapulmonary or i.v. routes. Data are means \pm S.E. for groups of three animals.

Table 2. Pharmacokinetic Data for BUD and DEX in Lung and Plasma

\begin{tabular}{|c|c|c|c|c|c|}
\hline \multirow{3}{*}{ Tissue } & \multirow{3}{*}{ Analyte } & \multicolumn{4}{|c|}{ Route of administration } \\
\hline & & \multicolumn{2}{|c|}{ Intrapulmonary } & \multicolumn{2}{|c|}{ Intravenous } \\
\hline & & $\begin{array}{c}A U C_{0-6 \mathrm{~h}} \\
(\mu \mathrm{M} \cdot \mathrm{h})\end{array}$ & $\begin{array}{l}t_{1 / 2} \\
(\mathrm{~h})\end{array}$ & $\begin{array}{c}A U C_{0-6 \mathrm{~h}} \\
(\mu \mathrm{M} \cdot \mathrm{h})\end{array}$ & $\begin{array}{l}t_{1 / 2} \\
\text { (h) }\end{array}$ \\
\hline \multirow[t]{2}{*}{ Lung } & DEX & 7.2 & 0.9 & 0.3 & 0.8 \\
\hline & BUD & 43.1 & 1.3 & & \\
\hline \multirow[t]{2}{*}{ Plasma } & DEX & 6.8 & 4.2 & 10.4 & 4.4 \\
\hline & BUD & N.D. & N.D. & & \\
\hline
\end{tabular}

$A U C_{0-6 \mathrm{~h}}$, the area under the curve from 0 to $6 \mathrm{~h} ; t_{1 / 2}$, elimination half-life; N.D., Not detected.

\section{DISCUSSION}

The present study demonstrated the following: (i) intrapulmonary treatment with BUD attenuates various inflammatory and fibrotic indices with minimal systemic adverse effects compared with DEX and (ii) pharmacokinetic analysis of both corticosteroids reveals that BUD exhibits slower clearance from the lung than DEX.

Current therapeutic strategies for treating lung fibrosis are aimed primarily at controlling the inflammatory process, often through the systemic administration of corticosteroids, which have been the first line of therapy for pulmonary fibrosis since the 1950s. ${ }^{17)}$ When high-dose corticosteroids are used in trials, significant and often irreversible adverse effects observed include weight gain, hyperglycemia, osteoporosis, avascular necrosis and gastrointestinal effects. ${ }^{4,17)}$

Inhaled corticosteroids (ICS) are the most common antiinflammatory treatment for asthma, rhinitis, cystic fibrosis and chronic obstructive pulmonary disease (COPD). ${ }^{6,7,18)}$ ICS treatment using inhalation devices provide relief of symptoms with few of the clinical side effects that are found with systemically administered corticosteroids. They are first-line therapy for control of asthma in all patients with persistent disease and have a central role in the treatment of asthma. ICS include BUD, fluticasone, beclomethasone and ciclesonide. They are delivered by inhalation devices, which can be divided into three categories: nebulizer, metered-dose inhaler (MDI) and dry-powder inhaler (DPI). ${ }^{19)}$ The results of a radiolabeled deposition study showed that approximately $20 \%$ of the emitted dose from a nebulizer and approximately $50 \%$ of the emitted dose of ciclesonide from a MDI were deposited in the lungs. ${ }^{20-22)}$

In this study, we found that ICS delivery to the lung induces optimal efficacy with minimal toxicity in a model of BLM-induced injury and fibrosis in rats compared to conventional and long-acting steroids; this result was based on the pharmacokinetic profiles of the antedrug. For toxic indexes by BUD and DEX in rats, we used thymus and spleen weights as a marker of glucocorticoid activity. ${ }^{23,24)}$ BUD has been shown to be retained in local-administrated tissue to a greater extent than conventional glucocorticosteroids, due to the intracellular formation and retention of BUD fatty acid esters. $^{25)}$ BUD is more rapidly metabolized by the liver than DEX. It has been reported that DEX was absorbed rapidly after intramuscular dosing (absorption half-life of $14 \mathrm{~min}$ ) with $86 \%$ bioavailability with a terminal half-life of $2.3 \mathrm{~h}$ after drug administration in rats. ${ }^{26)}$ DEX is rapidly transferred from administrated tissue to plasma with long terminal elimination half-life and low clearance.

Generally, the advantage of intrapulmonary delivery of corticosteroids is the following: (i) it delivers high drug concentrations directly to the disease site; (ii) it minimizes risks of systemic side effects and (iii) it can bypass the barriers to therapeutic efficacy such as first-pass metabolism in the liver. Although inhaled medications are widely accepted as the therapy for asthma and rhinitis, research on intrapulmonary drug delivery is still few, e.g. the chemotherapy of lung cancer and the administration of protein drugs like insulin. ${ }^{27)}$ To explore novel therapies for intractable pulmonary disease, drug action in disease lesions by Drug Delivery Systems (DDS) in combination with the advantage of using antedrugs 
will be beneficial. By avoiding undesirable systemic drug effects, our approach may improve the risk/benefit balance in the clinic.

In a review of ICS, Cerasoli illustrated the profile of the ideal ICS. The ideal ICS would have a larger therapeutic range than currently available agents, allowing doses to be increased without greatly increasing the frequency or severity of adverse events. The ideal ICS would possess the following pharmacokinetic properties to maximize efficacy and minimize side effects: high pulmonary deposition, conversion to an active metabolite, high receptor potency, high pulmonary retention, low oral bioavailability, extensive metabolism, and rapid elimination. ${ }^{7)}$ We think that the profile of the ideal ICS is very similar to the concept of the antedrug and the profile of the ideal aerosolized drugs for treating lung diseases. Small interfering RNA, gene therapy, antibodies and peptides possess these ICS-like profiles and would be the next generation of antedrugs. To date, various gene targets for pulmonary fibrosis have been identified as potential mediators of fibrosis and inflammation, including TGF- $\beta$, TNF$\alpha$, Interleukin- $\beta$ and Interleukin-13. ${ }^{28-30)}$ The ideal device for drug delivery to the lung would reduce the number of hurdles in the clinical development of novel therapeutics, such as next-generation antedrugs for lung disease.

In conclusion, intrapulmonary treatment with BUD attenuates various inflammatory and fibrotic indices with minimal systemic adverse effects compared with DEX. These findings suggest that the combination of intrapulmonary delivery and the antedrug approach is useful for the treatment of pulmonary fibrosis.

Acknowledgments We thank Dr. Satoru Takahashi (Division of Pathology, Nagoya City University Hospital) for expert advice on histological analysis and intrapulmonary administration, and Ms. Ritsuko Asai and Ms. Nana Kawaguchi of the Department of Pharmacoepidemiology, Kyoto University for their assistance with the animal experiments.

\section{REFERENCES}

1) Katzenstein A. L. A., Myers J. L., Am. J. Respir. Crit. Care Med., 157, 1301-1315 (1998).

2) Luppi F., Cerri S., Beghe B., Fabbri L. M., Richeldi L., Respir. Med., 98, 1035-1044 (2004).

3) du Bois R. M., Nat. Rev. Drug Discov., 9, 129-140 (2010).

4) Flaherty K. R., Toews G. B., Lynch J. P. 3rd, Kazerooni E. A., Am. J.
Med., 110, 278-282 (2001).

5) Lee H. J., Soliman M. R., Science, 215, 989—991 (1982).

6) Mygind N., Andersson M., Acta Otolaryngol., 126, 1022-1029 (2006).

7) Cerasoli F. Jr., Chest, 130, 54S-64S (2006).

8) Campieri M., Ferguson A., Doe W., Persson T., Nilsson L. G., Gut, 41, 209-214 (1997).

9) Yokohira M., Takeuchi H., Yamakawa K., Saoo K., Matsuda Y., Zeng Y., Hosokawa Y., Imaida K., Exp. Toxicol. Pathol., 58, 211-221 (2007).

10) Yokohira M., Kuno T., Yamakawa K., Hashimoto N., Ninomiya F., Suzuki S., Saoo K., Imaida K., J. Toxicol. Pathol., 22, 1-10 (2009).

11) Yao H. W., Zhu J. P., Zhao M. H., Lu Y., Respiration, 73, 236-242 (2006).

12) Iglesias Y., Fente C. A., Vázquez B., Franco C., Cepeda A., Mayo S., Gigosos P. G., J. Agric. Food Chem., 47, 4275-4279 (1999).

13) Chanoine F., Grenot C., Heidmann P., Junien J. L., Drug Metab. Dispos., 19, 546-553 (1991).

14) Gong L. K., Li X. H., Wang H., Zhang L., Chen F. P., Cai Y., Qi X. M., Liu L. L., Liu Y. Z., Wu X. F., Huang C. G., Ren J., J. Ethnopharmacol., 96, 537-544 (2005).

15) Günther A., Lübke N., Ermert M., Schermuly R. T., Weissmann N., Breithecker A., Markart P., Ruppert C., Quanz K., Ermert L., Grimminger F., Seeger W., Am. J. Respir. Crit. Care Med., 168, 1358-1365 (2003).

16) Huaux F., Liu T., McGarry B., Ullenbruch M., Phan S. H., J. Immunol., 170, 2083-2092 (2003)

17) Walter N., Collard H. R., King T. E. Jr., Proc. Am. Thorac. Soc., 3, $330-338$ (2006)

18) Barnes P. J., Stockley R. A., Eur. Respir. J., 25, $1084-1106$ (2005).

19) Labiris N. R., Dolovich M. B., Br. J. Clin. Pharmacol., 56, 600-612 (2003).

20) Laube B. L., Geller D. E., Lin T. C., Dalby R. N., Diener-West M., Zeitlin P. L., Respir. Care, 50, 1438-1444 (2005).

21) Newman S., Salmon A., Nave R., Drollmann A., Respir. Med., 100 $375-384$ (2006).

22) Usmani O. S., Biddiscombe M. F., Barnes P. J., Am. J. Respir. Crit. Care Med., 172, 1497-1504 (2005).

23) Chen Z., Vaughn D. A., Blakely P., Fanestil D. D., J. Am. Soc. Nephrol., 5, 1361-1368 (1994).

24) Keil D. E., Luebke R. W., Pruett S. B., Int. J. Immunopharmacol., 17, 157-166 (1995).

25) Miller-Larsson A., Jansson P., Runström A., Brattsand R., Am. J. Respir. Crit. Care Med., 162, 1455-1461 (2000).

26) Samtani M. N., Jusko W. J., Biopharm. Drug Dispos., 26, 85-91 (2005).

27) Labiris N. R., Dolovich M. B., Br. J. Clin. Pharmacol., 56, 588-599 (2003).

28) Fichtner-Feigl S., Strober W., Kawakami K., Puri R. K., Kitani A., Nat. Med., 12, 99-106 (2006).

29) Ask K., Martin G. E., Kolb M., Gauldie J., Proc. Am. Thorac. Soc., 3, 389-393 (2006).

30) Moeller A., Ask K., Warburton D., Gauldie J., Kolb M., Int. J. Biochem. Cell Biol., 40, 362-382 (2008). 